\title{
Multimodal Optical Coherence Tomography: Imaging of Blood and tymphatic Vessels of the Vulva
}

DOI: $10.17691 / \mathrm{stm} 2019.11 .4 .03$

Received September 17, 2019

M.A. Sirotkina, PhD, Head of Scientific Laboratory of High-Resolution Microscopy

and Gene Technologies, Institute of Experimental Oncology and Biomedical Technologies';

A.L. Potapov, Student?;

N.N. Vagapova, Gynecologist2:

1.K. Safonov, Clinical Resident';

D.A. Karashtin, PhD, Researcher, Laboratory of Atmospheric Electricity3;

L.A. Matveev, PhD, Senior Researcher, Laboratory of Wave Methods

for Studying Structurally Inhomogeneous Media3;

S.G. Radenska-Lopovok, MD, DSc, Professor, A.I. Strukov Department of Pathological Anatomy4;

A.A. Timakova, Student';

S.S. Kuznetsov, MD, DSc, Professor, Head of Pathological and Anatomical Department²;

E.V. Zagaynova, MD, DSc, Professor of the Russian Academy of Sciences, Director of the Institute

of Experimental Oncology and Biomedical Technologies';

I.A. Kuznetsova, MD, DSc, Associate Professor, Department of Obstetrics and Gynecology';

N.D. Gladkova, MD, DSc, Professor, Head of the Scientific Laboratory of Optical Coherence Tomography,

Institute of Experimental Oncology and Biomedical Technologies ${ }^{1}$

1Privolzhsky Research Medical University, 10/1 Minin and Pozharsky Square, Nizhny Novgorod,

603005, Russia;

${ }^{2}$ N.A. Semashko Nizhny Novgorod Regional Clinical Hospital, 190 Rodionova St., Nizhny Novgorod,

603126, Russia;

${ }^{3}$ Federal Research Center Institute of Applied Physics, Russian Academy of Sciences, 46 Ulyanova St.,

Nizhny Novgorod, 603950, Russia;

${ }^{4}$ I.M. Sechenov First Moscow State Medical University (Sechenov University), 8/2 Trubetskaya St., Moscow, 119991, Russia

The aim of the study was to test the method of optical coherence tomography (OCT) for its ability to obtain images of blood and lymphatic vessels of the vulva.

Materials and Methods. The study was performed using a multimodal optical coherence tomography device developed in the Institute of Applied Physics of the Russian Academy of Sciences (Nizhny Novgorod). In this setup, 3D images of $3.4 \times 3.4 \times 1.25 \mathrm{~mm}$ are created within $26 \mathrm{~s}$. OCT angiography and OCT lymphangiography are based on the speckle structure analysis. Visualization of blood and lymphatic vessels does not require any additional contrast agents.

A histological study of vulvar biopsy samples from two locations was performed in 3 patients without vulvar pathology and in 5 patients with vulvar lichen sclerosus; the results were then compared with 3D OCT images obtained at the same locations.

Results. Using the multimodal OCT, we found that normal mucous membrane of the vulva had a well-developed network of blood and lymphatic vessels; their diameters were increasing with depth. In the subepithelial zone, only blood capillary loops could be seen, whereas lymphatic capillaries became detectable starting from a depth of $170 \mu \mathrm{m}$ (submucosa layer).

In the case of vulvar lichen sclerosus, the density of the blood vessel network decreases dramatically and the capillary loops in the subepithelial zone disappear. Lymphatic vessels become detectable at a depth of $300 \mu \mathrm{m}$, and only few thin vessels can be detected at depths of 300 to $600 \mu \mathrm{m}$. The results suggest that the presentation of blood and lymphatic vessels depends on the state of the vulvar connective tissue. Specifically, the number of blood and lymphatic vessels is reduced in areas of hyalinosis and sclerosis of collagen fibers.

Conclusion. For the first time, using multimodal OCT, an in vivo study of blood and lymphatic vessels of the vulvar mucosa was performed on normal and lichen sclerosus-affected vulva; the obtained OCT images were then compared with histological images of the same tissues. The OCT method allows one to diagnose a zone of hyalinosis and sclerosis of collagen fibers characterized by an abnormally low number of blood and lymphatic vessels.

Key words: OCT angiography; OCT lymphangiography; vulva; lichen sclerosus; OCT microcirculation; blood vessels; lymphatic vessels.

Corresponding author: Marina A. Sirotkina, e-mail: sirotkina_m@mail.ru 


\section{Introduction}

Lichen sclerosus is a chronic inflammatory of skin and mucous disorder of genital and extragenital localization. This is a debilitating disease manifested in itching, pain, dysuria, urination problems, dyspareunia, and sexual dysfunctions in women [1]. The etiology of lichen sclerosus is still not clear, but recent data point to the autoimmune etiology of the disease in genetically predisposed individuals. Thus, in a study of Oyama et al. [2], circulating autoantibodies to ECM1 (extracellular matrix protein 1) were found in about $75 \%$ of patients with lichen sclerosus (versus $7 \%$ in control). Confirmation of these data can be found elsewhere [3].

Normally, ECM1 is expressed in various components of the skin, including all active epidermis layers, blood vessel walls and collagen fibers of the dermis, thereby contributing to the maintenance of the overall skin structure. The ECM1 is known to be involved in the control of keratinocyte differentiation of the epidermis; it plays a role in the structural organization of the dermis, where it binds to perlikan, matrix metalloproteinase 9 and fibulin; it also stimulates the process of angiogenesis and proliferation of endothelial cells [4].

Dysfunction of ECM1 in lichen sclerosus occurs due to an autoimmune reaction and leads to hyperkeratosis and/or atrophy of the epithelium, homogenization, sclerosis, and hyalinosis of collagen fibers in the dermis, and damage to the endothelium leading to increased permeability of the vascular wall [4]. In lichen sclerosus, a decrease in ECM1 expression in blood vessels occurs against the background of increased synthesis of type $\mathrm{V}$ collagen, which causes hyalinisation of the subepithelial zone [5]. It is important to note that the hyalinization of blood capillaries and collagen fibers of the papillary dermis is a classic sign of lichen sclerosus; back in 1986, this symptom was proposed as a marker of severity of this disease [6].

In a number of studies, changes in blood vessels during the development of vulvar lichen sclerosus were studied in detail [7-9]. Thus, in [8], several phenomena were discovered using electron microscopy and immunohistochemical staining of biopsy material: a) damage to endothelial cells of blood vessels due to the detachment of pericytes from the endothelium; b) disruption of blood vessel architectonics; and c) disappearance of capillaries from the papillary dermis.

The data about the role of lymphatic vessels in the pathogenesis of lichen sclerosus is lack. In healthy skin, lymphatic vessels are blind-ending vessels originating in the interstitial space of the dermal papillae. Lymphatic capillaries range from 30 to $80 \mu \mathrm{m}$ in diameter; their thin walls consist of one layer of endothelial cells shaped as an oak leaf [10]. The lymphatic vasculature plays a role in the mechanisms of local inflammation and immunity; its dysfunction can lead to uncontrolled inflammation. Lymphatic insufficiency makes the skin and/or mucous membrane vulnerable to infection, inflammation and even malignancy [10-12].

The in vivo diagnosis of vulvar dystrophic diseases is complicated by the lack of informative and objective imaging methods. To rule out malignancy, vulvoscopy is usually performed to detect atypical epithelium. A serious limitation of vulvoscopy is its sensitivity to the epithelium thickness, which is uneven in different parts of the vulva, varies from patient to patient and can be masked with stratum corneum on the surface. Until now, there are no generally accepted vulvoscopic signs of vulvar dystrophy; therefore, the diagnostic value of vulvoscopy is debatable [13]. The gold standard for diagnosing vulvar dystrophy is still the histological analysis of biopsy samples.

Multimodal optical coherence tomography (OCT) is a non-invasive method for obtaining images of biological tissues with a high spatial resolution (10-15 $\mu \mathrm{m})$ up to a depth of $1.5 \mathrm{~mm}[14,15]$. Using OCT, one can evaluate both the general structure of biological tissue (number and thickness of its layers), the state of the connective tissue, and microcirculation (blood flow and lymph flow) in real time. This approach has already proven itself in solving a number of tasks, e.g., visualizing the microcirculation in the oral mucosa [16] and monitoring the vascular response to treatment $[17,18]$.

OCT lymphangiography is based on the analysis of spatio-temporal speckle statistics in areas of interest; this statistics is then compared with the expected one [19, 20]. In the OCT, microcirculation in blood and lymphatic vessels is visualized without using any contrast agents.

We were unable to find reports on in vivo studies of blood and lymphatic vessels in the vulvar tissue. Therefore, the aim of this research was to assess the feasibility of using multimodal OCT for studying vulvar blood and lymphatic vessels in normal tissues and those affected with lichen sclerosus.

\section{Materials and Methods}

Characterization of patients. The study involved patients undergoing treatment in the Department of Gynecology of the N.A. Semashko Nizhny Novgorod Regional Clinical Hospital. Eight patients were divided into two groups. In group 1, there were three women (without vulvar diseases) hospitalized for vaginal wall prolapse. Their average age was 49 years old (30-65 years old). Group 2 included 5 women with vulvar lichen sclerosus; they complained of dry vulvar mucosa and severe itching, especially at night. The average age of women in this group was 57.2 years old (46-68 years old). The OCT study was conducted in patients without pronounced hyperkeratosis, which prevents the light penetration.

All studies were approved by the Ethics Committee of the Privolzhsky Research Medical University. OCT studies were performed with informed consent of the patients.

Multimodal OCT. The study of vulvar blood and lymphatic vessels was performed by means of the in 
vivo multimodal OCT method without using contrast agents. The spectral OCT device was developed at the Institute of Applied Physics, Russian Academy of Sciences (Nizhny Novgorod) [21, 22]. The source of light in the OCT is a superluminescent diode with a central wavelength of $1310 \mathrm{~nm}$ and a spectrum width of $100 \mathrm{~nm}$. The longitudinal resolution is $10 \mu \mathrm{m}$, the lateral resolution is $15 \mu \mathrm{m}$ (the full beam width is at the half of its maximum). In this setup, 3D images of $3.4 \times 3.4 \times 1.25 \mathrm{~mm}$ are created within $26 \mathrm{~s}$. The OCT setup is equipped with a flexible fiber-optic probe, which ends with a "pencil" type lens (length $-15 \mathrm{~cm}$, diameter $-1 \mathrm{~cm}$ ) and is intended for contact examination of a tissue. OCT angiography and OCT lymphangiography are based on the analysis of the speckle structure [23, 24].

For each patient, a 3D OCT data set was obtained from two areas (right and left) located in the central zone at the border of the inner and middle vulvar rings [13]. For each point, we then obtained 2D images of the tissue in-depth cross section - a structural OCT image. From this image, the number of layers, thickness of the epithelium, the presence of keratin masses, and the OCT signal from the connective tissue were estimated.
En-face images of blood and lymphatic vessels at various depths were also obtained.

Histological analysis. Morphology of the vulvar tissues was studied using histological microscopy after staining with hematoxylin and eosin and picrofuchsin according to Van Gieson. Verification of lymphatic vessels in the vulvar tissue was performed by immunohistochemistry using antibodies to Podoplanin - the lymphatic endothelial cell marker. Histological preparations were evaluated using a Leica DM 2500 microscope (Leica Biosystems, Germany) with a DFC 245 C digital video camera in transmitted light. Biopsy material was taken immediately after the OCT observation had been completed. The biopsy accuracy was ensured by marking the boundaries of the OCT measurement.

\section{Results and Discussion}

OCT imaging of normal vulvar tissue. Normally, the skin and mucous membrane of the vulva are uniformly pale-pink. In OCT images, a layered structure is clearly seen (Figure $1(a),(b))$. The upper layer with low signal
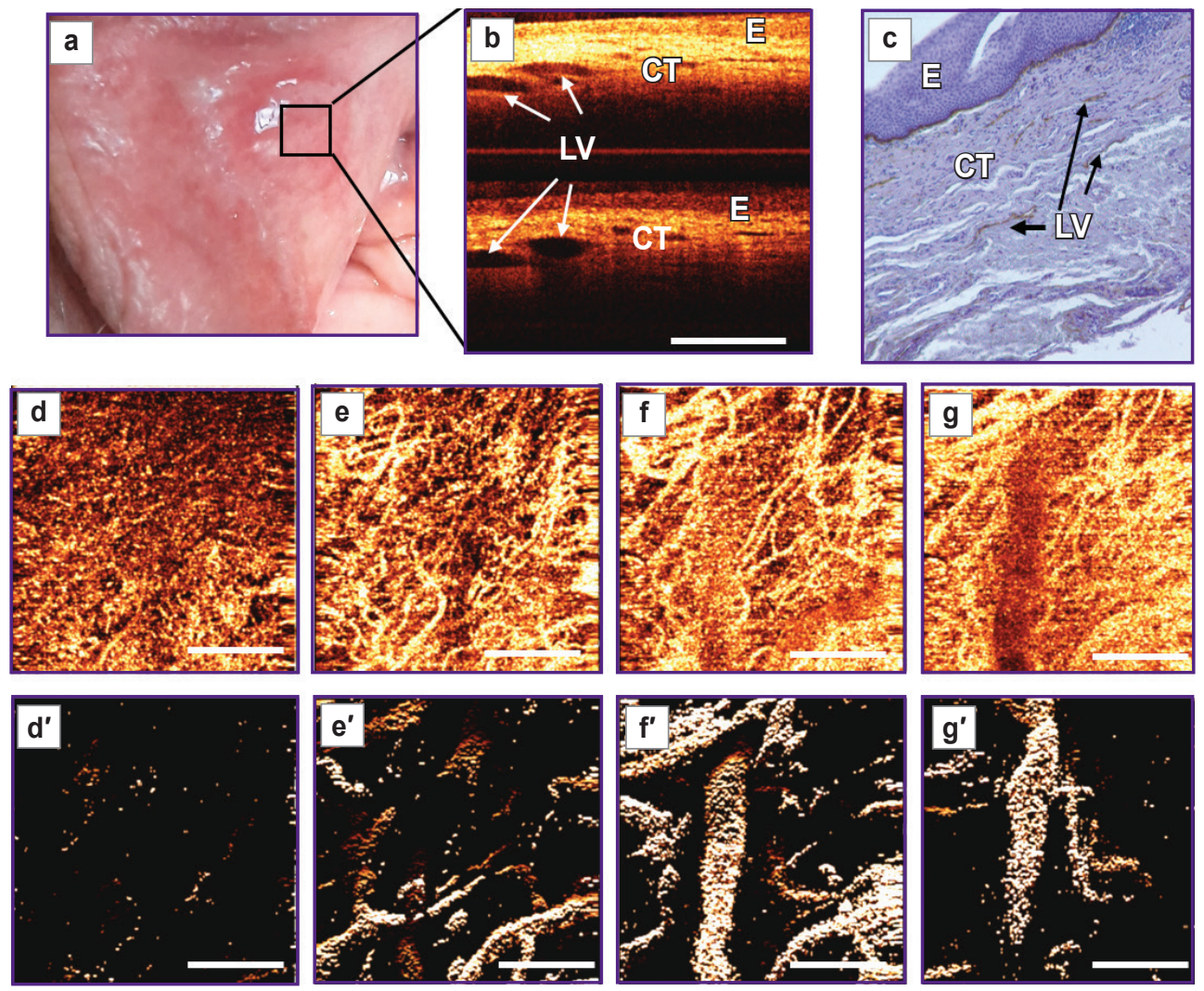

Figure 1. Patient K., 67 years old; mucous membrane of the vulva without pathology:

(a) area where the OCT study and biopsy were performed; (b) structural OCT image (B-scan);

(c) immunohistochemical staining of lymphatic vessels using antibodies to Podoplanin; (d) and

(d') blood and lymphatic vessels (en-face OCT images) in the subepithelium zone at a depth of up to $170 \mu \mathrm{m}$ from the surface of the vulva; (e) and (e') blood and lymphatic vessels at a depth of 170-300 $\mu \mathrm{m}$; (f) and (f') blood and lymphatic vessels at a depth of 300-450 $\mu \mathrm{m}$; $(\mathrm{g})$ and $\left(\mathrm{g}^{\prime}\right)$ blood and lymphatic vessels at a depth of $450-600 \mu \mathrm{m}$; bar size is $1 \mathrm{~mm}$. Arrows indicate lymphatic vessels. E - epithelium; CT — connective tissue; LV — lymphatic vessels 
amplitude corresponds to the epithelium. The thickness of the epithelium is even, the border with the underlying connective tissue is contrasting. The OCT signal from the connective tissue is high; it attenuates rapidly with depth.

Elongated inclusions with a low OCT signal, which are visible in the connective tissue, correspond to lymphatic vessels upon immunohistochemical staining (Figure 1 (c)). In OCT angiographic images (Figure 1 (d)-(g)), a dense network of blood vessels is visualized. In the subepithelial layer, at a depth of about $170 \mu \mathrm{m}$ from the tissue surface, one can see mostly blood capillary loops (see Figure 1 (d)). In deeper layers of the connective tissue, at depths of 170 to $600 \mu \mathrm{m}$, a network of arterioles and venules appears. The vessel diameter increases with depth (see Figure 1 (e)-(g)). The OCT angiography is sensitive to flows in vessels with micrometer-size diameters (subresolvable capillaries) and wider; the minimum capillary size required for creating an OCT angiogram is close to the resolution capacity of the OCT system $(15 \mu \mathrm{m})$.
Unlike blood vessels, lymphatic vessels form a less dense network of larger vessels (Figure $\left.1\left(d^{\prime}\right)-\left(g^{\prime}\right)\right)$. The largest lymphatic vessels are located in the connective tissue at a depth of 300-450 $\mu \mathrm{m}$ (see Figure 1 ( $\left.\mathrm{f}^{\prime}\right)$ ). It is important to note that in the subepithelial layer, at a depth of up to $170 \mu \mathrm{m}$ from the tissue surface, lymphatic vessels are not visualized by OCT-lymphangiography (see Figure $1\left(d^{\prime}\right)$ ). This can be explained by the fact that lymphatic capillaries have not yet been formed in this area. Here, the lymphatic system is represented by so-called prelimphatics, i.e., tissue gaps along bundles of collagen fibers [25], with the size below the OCT resolution capacity.

OCT imaging of vulvar tissue in lichen sclerosus. This type of lichen is clinically manifested by porcelainwhite papules or plaques, which are accompanied by redness, hyperkeratosis, cracks, and erosion (Figure 2 (a)). In the examined patients, it spread to the labia minora, labia majora, clitoris, and adjacent areas.

In the OCT images, vulvar tissues affected by lichen sclerosus retain a layered structure, but the epithelium
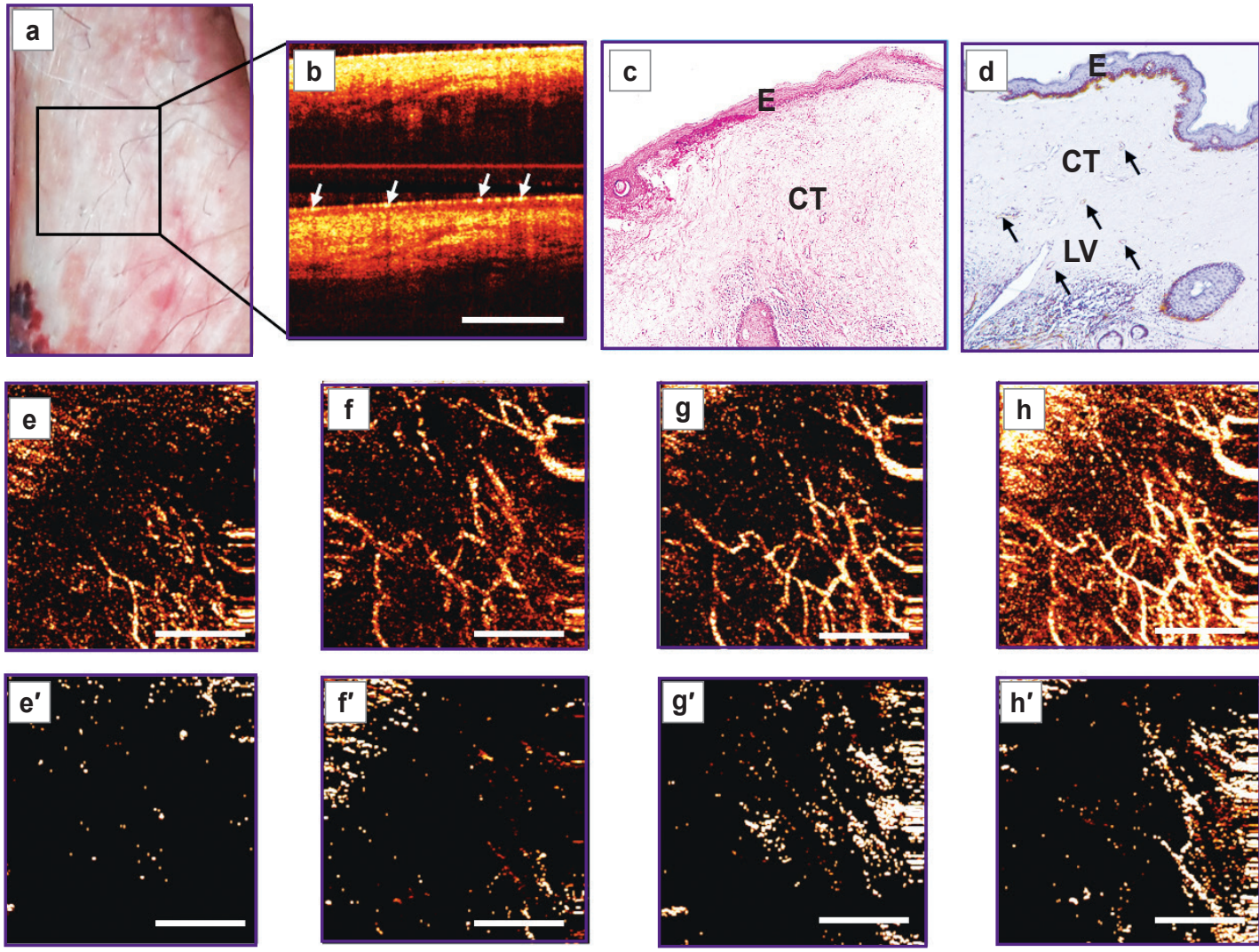

Figure 2. Patient K., 58 years old. Diagnosis is vulvar lichen sclerosus:

(a) area where the study was performed; (b) structural OCT image (B-scan); (c) histological preparation stained with hematoxylin and eosin; (d) immunohistochemical staining of lymphatic vessels using antibodies to Podoplanin; (e) and (e') blood and lymphatic vessels (en-face OCT images) in the subepithelium zone at a depth of up to $170 \mu \mathrm{m}$ from the surface of the vulva; $(\mathrm{f})$ and $\left(\mathrm{f}^{\prime}\right)$ blood and lymphatic vessels at a depth of 170-300 $\mu \mathrm{m} ;(\mathrm{g})$ and $\left(\mathrm{g}^{\prime}\right)$ blood and lymphatic vessels at a depth of 300-450 $\mu \mathrm{m}$; $(\mathrm{h})$ and $\left(\mathrm{h}^{\prime}\right)$ blood and lymphatic vessels at a depth of $450-600 \mu \mathrm{m}$; bar size is $1 \mathrm{~mm}$. E - epithelium; CT — connective tissue; LV - lymphatic vessels. White arrows indicate keratin masses accumulated on the surface of the epithelium, black arrows - lymphatic vessels 
becomes very thin (atrophic) with an even thickness (Figure 2 (b)). According to the histological data, the thickness of the epithelium is $35-55 \mu \mathrm{m}$. Its border with the connective tissue is less contrasted than normally. Bright spots indicative of hyperkeratosis appear on the surface of the epithelium (Figure 2 (c)). In B-scans, there are very few elongated inclusions in the connective tissue (lymphatic vessels).

In histological sections, the connective tissue looks changed. In the foci of chronic inflammation, sclerotic collagen fibers are obtained (see Figure 2 (c)). Sclerosis begins immediately under the epithelium and spreads deeper (in our observations, from 145 to $880 \mu \mathrm{m})$. In some cases, foci of hyalinosis are noted in the sclerotized stroma of the subepithelial zone. In areas of sclerosis and hyalinosis, there are fewer blood vessels in the subepithelial zone; in addition, vascular and perivascular sclerosis can be seen in almost all blood vessels. There are isolated if any lymphatic vessels in this area (Figure 2 (d)). In deep layers of the connective tissue, where the collagen fibers remain intact (under the zone of sclerosis), the blood and lymphatic vessels are unchanged and present in large numbers.

In OCT angiographic images of lichen sclerosus at all depths tested, the blood vessel network is much less developed compared to the norm (Figure 2 (e)-(h)). In the subepithelial zone, at a depth of $170 \mu \mathrm{m}$ from the surface, the blood capillary loops characteristic of the normal mucous membrane, disappear. There are either no capillaries or just fragmented vessels in this zone (see Figure $2(\mathrm{e})$ ). It is known that OCT angiography is able to visualize only vessels with active blood flow [26]. Vessels with critically low or zero blood flow are no longer visible. Perivascular sclerosis of the blood vessel walls, leading to a significant decrease in the vessel lumen, in all likelihood, is the cause of poor visualization of blood vessels in OCT angiographic images.

In the OCT lymphangiographic images of lichen sclerosus, at a depth of up to $300 \mu \mathrm{m}$, lymphatic vessels characteristic of the normal vulvar mucosa, are not seen (Figure $\left.2\left(e^{\prime}\right),\left(f^{\prime}\right)\right)$. Further, at depths of 300 to $600 \mu \mathrm{m}$, in some cases very thin and fragmented lymphatic vessels can be found (Figure $2\left(g^{\prime}\right)$, $\left.\left(h^{\prime}\right)\right)$. The above changes are caused by gross abnormalities of the connective tissue - sclerosis and hyalinosis of collagen fibers.

In summary, OCT structure imaging of the multilayer vulvar tissue combined with OCT angiography and lymphangiography, allows one to conduct an in vivo (without contrast agents) real-time visualization of the epithelium, connective tissue, and networks of blood and lymphatic vessels. This OCT modality makes it possible to assess the tissue structures at different depths and thereby detect damage to the connective tissue affected by lichen sclerosus as well as monitor the tissue state during treatment.

\section{Conclusion}

For the first time, the multimodal OCT method has been used to study microcirculation (blood flow and lymph flow) at different depths of the vulvar mucosa under normal and lichen sclerosus conditions. The results suggest that the architectonics of blood and lymphatic vasculature depends on the state of the connective tissue. Thus, sclerosis and hyalinosis of the subepithelial layer lead to a dramatically decrease in density of the blood vessel network and almost complete disappearance of lymphatic vessels, which further impairs the tissue trophic.

Financial support. The OCT study of vulvar tissue was supported by the Russian Science Foundation, agreement No.19-75-10084. The development of OCTlymphangiography based on the analysis of speckle parameters was supported by Russian President grant for young scientists No.MK-3416.2018.2 (L.A. Matveev).

Conflict of interest. The authors have no conflict of interest.

\section{References}

1. Tasker G.L., Wojnarowska F. Lichen sclerosus. Clin Exp Dermatol 2003; 28(2): 128-133, https://doi.org/10.1046/j.13652230.2003.01211.x.

2. Oyama N., Chan I., Neill S.M., South A.P., Wojnarowska F., Kawakami Y., D'Cruz D., Mepani K., Hughes G.J., Bhogal B.S., Kaneko F., Black M.M., McGrath J.A. Development of antigen-specific ELISA for circulating autoantibodies to extracellular matrix protein 1 in lichen sclerosus. J Clin Invest 2004; 113(11): 1550-1559, https://doi.org/10.1172/jci200420373.

3. Olek-Hrab K., Jenerowicz D., Osmola-Mańkowska A., Polańska A., Teresiak-Mikołajczak E., Silny W., Adamski Z. Selected vulvar dermatoses. Ginekol Pol 2013; 84(11): 959 965, https://doi.org/10.17772/gp/1667.

4. Oyama N., Merregaert J. The extracellular matrix protein 1 (ECM1) in molecular-based skin biology. In: Farage M., Miller K., Maibach H. (editors). Textbook of aging skin. Springer, Berlin, Heidelberg; 2015; p. 1-20, https://doi. org/10.1007/978-3-642-27814-3_8-2.

5. Godoy C.A., Teodoro W.R., Velosa A.P., Garippo A.L., Eher E.M., Parra E.R., Sotto M.N., Capelozzi V.L. Unusual remodeling of the hyalinization band in vulval lichen sclerosus by type $\mathrm{V}$ collagen and ECM 1 protein. Clinics (Sao Paulo) 2015; 70(5): 356-362, https://doi.org/10.6061/ clinics/2015(05)09.

6. Hewitt J. Histologic criteria for lichen sclerosus of the vulva. J Reprod Med 1986; 31(9): 781-787.

7. Kowalewski C., Kozłowska A., Chan I., Górska M., Woźniak K., Jabłońska S., McGrath J.A. Three-dimensional imaging reveals major changes in skin microvasculature in lipoid proteinosis and lichen sclerosus. J Dermatol Sci 2005; 38(3): 215-224, https://doi.org/10.1016/j.jdermsci.2005.01.012.

8. Li Y.Z., Wu Y., Zhang Q.H., Wang Y., Zhen J.H., Li S.L. Hypoxia-ischaemia is involved in the pathogenesis of vulvar lichen sclerosus. Clin Exp Dermatol 2009; 34(8): e531-e536, https://doi.org/10.1111/j.1365-2230.2009.03571.x. 
9. van der Avoort I.A., van der Laak J.A., Otte-Höller I., van de Nieuwenhof H.P., Massuger L.F., de Hullu J.A., van Kempen L.C. The prognostic value of blood and lymph vessel parameters in lichen sclerosus for vulvar squamous cell carcinoma development: an immunohistochemical and electron microscopy study. Am J Obstet Gynecol 2010; 203(2): 167.e1-167.e8, https://doi.org/10.1016/j. ajog.2010.02.002.

10. Alitalo K. The lymphatic vasculature in disease. Nat Med 2011; 17(11): 1371-1380, https://doi.org/10.1038/nm.2545.

11. Ruocco E., Puca R.V., Brunetti G., Schwartz R.A., Ruocco V. Lymphedematous areas: privileged sites for tumors, infections, and immune disorders. Int J Dermatol 2007; 46(6): 662, https://doi.org/10.1111/j.1365-4632.2007.03244.x.

12. Lund A.W., Medler T.R., Leachman S.A., Coussens L.M. Lymphatic vessels, inflammation, and immunity in skin cancer. Cancer Discov 2016; 6(1): 22-35, https://doi.org/10.1158/21598290.cd-15-0023.

13. Harni V., Babic D., Barisic D. "Three rings vulvoscopy" a new approach to the vulva. In: Watson L. (editor). Cryosurgery and colposcopy. Nova Science Publishers, Inc.; 2016; p. 146-153.

14. Gelikonov G.V., Gelikonov V.M. New approach to cross-polarized optical coherence tomography based on orthogonal arbitrarily polarized modes. In: Proc. SPIE 6429, Coherence Domain Optical Methods and Optical Coherence Tomography in Biomedicine XI, 64290L. SPIE; 2007, https:// doi.org/10.1117/12.704015.

15. Husvogt L., Ploner S., Maier A. Optical coherence tomography. In: Maier A., Steidl S., Christlein V., Hornegger J. (editors). Medical imaging systems. Lecture notes in computer science. Vol. 11111. Springer, Cham; 2018; p. 251-261, https:// doi.org/10.1007/978-3-319-96520-8_12.

16. Maslennikova A.V., Sirotkina M.A., Moiseev A.A., Finagina E.S., Ksenofontov S.Y., Gelikonov G.V., Matveev L.A., Kiseleva E.B., Zaitsev V.Y., Zagaynova E.V., Feldchtein F.I., Gladkova N.D., Vitkin A. In-vivo longitudinal imaging of microvascular changes in irradiated oral mucosa of radiotherapy cancer patients using optical coherence tomography. Sci Rep 2017; 7(1): 16505, https://doi. org/10.1038/s41598-017-16823-2.

17. Sirotkina M.A., Matveev L.A., Shirmanova M.V., Zaitsev V.Y., Buyanova N.L., Elagin V.V., Gelikonov G.V., Kuznetsov S.S., Kiseleva E.B., Moiseev A.A., Gamayunov S.V., Zagaynova E.V., Feldchtein F.I., Vitkin A., Gladkova N.D. Photodynamic therapy monitoring with optical coherence angiography. Sci Rep 2017; 7(1): 41506, https://doi. org/10.1038/srep41506.

18. Sirotkina M.A., Moiseev A.A., Matveev L.A., Zaitsev V.Y., Elagin V.V., Kuznetsov S.S., Gelikonov G.V., Ksenofontov S.Y., Zagaynova E.V., Feldchtein F.I., Gladkova N.D., Vitkin A.
Accurate early prediction of tumour response to PDT using optical coherence angiography. Sci Rep 2019; 9(1): 6492, https://doi.org/10.1038/s41598-019-43084-y.

19. Leitgeb R., Hitzenberger C.K., Fercher A.F. Performance of fourier domain vs. time domain optical coherence tomography. Opt Express 2003; 11(8): 889-894, https://doi.org/10.1364/oe.11.000889.

20. Matveev L.A., Demidov V.V., Sovetsky A.A., Moiseev A.A., Matveyev A.L., Gelikonov G.V., Zaitsev V.Y., Vitkin A. OCT-based label-free 3D mapping of lymphatic vessels and transparent interstitial-fluid-filled dislocations. In: International conference laser optics (ICLO). IEEE; 2018; p. 512, https://doi.org/10.1109/lo.2018.8435727.

21. Shilyagin P.A., Gelikonov G.V., Gelikonov V.M., Moiseev A.A., Terpelov D.A. Achromatic registration of quadrature components of the optical spectrum in spectral domain optical coherence tomography. Quantum Electronics 2014; 44(7): 664, https://doi.org/10.1070/ qe2014v044n07abeh015465.

22. Shilyagin P.A., Ksenofontov S.Y., Moiseev A.A., Terpelov D.A., Matkivsky V.A., Kasatkina I.V., Mamaev Y.A., Gelikonov G.V., Gelikonov V.M. Equidistant recording of the spectral components in ultra-wideband spectral-domain optical coherence tomography. Radiophysics and Quantum Electronics 2018; 60(10): 769-778, https://doi.org/10.1007/s11141-0189845-z.

23. Matveev L.A., Demidov V., Sirotkina M.A., Karashtin D.A., Moiseev A.A., Popov I., Sovetsky A.A., Matveyev A.L., Demidova O., Gelikonov G.V., Flueraru C., Zagaynova E.V., Gladkova N.D., Zaitsev V.Y., Vitkin I.A. OCT lymphangiography based on speckle statistics evaluation. In: Proc. SPIE 11065, Saratov Fall Meeting 2018: Optical and Nano-Technologies for Biology and Medicine, 1106502. SPIE; 2019, https://doi.org/10.1117/12.2523291.

24. Moiseev A.A., Ksenofontov S., Sirotkina M., Kiseleva E., Gorozhantseva M., Shakhova N., Matveev L., Zaitsev V., Matveyev A., Zagaynova E., Gelikonov V., Gladkova N., Vitkin A., Gelikonov G. Optical coherence tomography-based angiography device with real-time angiography B-scans visualization and hand-held probe for everyday clinical use. J Biophotonics 2018; 11(10): e201700292, https://doi.org/10.1002/jbio.201700292.

25. Borodin Yu.I. Lymphatic system and ageing Fundamental'nye issledovaniya 2011; 5: 11-15.

26. Matveev L.A., Zaitsev V.Y., Gelikonov G.V., Matveyev A.L., Moiseev A.A., Ksenofontor S.Y., Gelikonov V.M., Sirotkina M.A., Gladkova N.D., Demidov V., Vitkin A. Hybrid M-mode-like OCT imaging of three-dimensional microvasculature in vivo using reference-free processing of complex valued B-scans. Opt Lett 2015; 40(7): 1472-1475, https://doi.org/10.1364/ol.40.001472. 